\title{
ALMOST PERIODIC COMPACTIFICATIONS
}

\author{
BY KAREL DELEEUW AND IRVING GLICKSBERG ${ }^{1}$
}

Communicated by J. L. Kelley, February 13, 1959

1. Introduction. Let $B$ be a Banach space and $S$ a uniformly bounded semigroup of operators on $B$; i.e., a family $S$ of operators closed under the usual multiplication of operators and satisfying $\|T\| \leqq K$ for some $K$ and all $T$ in $S . S$ will be called almost periodic (a.p.) if each orbit is conditionally compact (that is, has compact closure) in the norm topology of $B$ and will be called weakly almost periodic (w.a.p.) if each orbit is conditionally compact in the weak topology of $B$. In either situation, the closure $\bar{S}$ of $S$ in the algebra of bounded operators on $B$ supplied with the weak operator topology is a compact semigroup. Multiplication in $\bar{S}$ is jointly continuous in the a.p. case while only separately continuous in the w.a.p. case.

The purpose of this note is to announce some results which have in common the exploitation of this "compactification" $\bar{S}$ of $S$.

In $\S 2$ we extend the results of Jacobs given in [8] and [9]. In $\S 3$ the theory of weakly almost periodic functions due to Eberlein (see [3]) is extended to certain topological semigroups by constructing a compactification that plays the same role in the theory of weakly almost periodic functions as does the Bohr compactification (see [1] and [12]) in the theory of almost periodic functions on groups. In $\S 4$ we study almost periodic functions on semigroups. Our class of functions is wider then that studied by Maak in [10] and in the case of the half-line is identical with the class of functions studied by Fréchet in [6].

TERMINOLOGY. By a topological semigroup we shall mean a semigroup with identity supplied with a Hausdorff topology in which the maps $\tau \rightarrow \sigma \cdot \tau$ and $\tau \rightarrow \tau \cdot \sigma$ are continuous for each $\sigma$ in $S$; multiplication is not assumed to be jointly continuous. When we use the term topological group we will mean, as usual, a group supplied with a Hausdorff topology in which multiplication is jointly continuous and inversion is continuous. If $S$ is a topological semigroup, we shall denote by $C(S)$ the Banach space of all complex valued bounded continuous functions on $S$, supplied with the norm defined by

$$
\|f\|=\sup _{\sigma \in S}|f(\sigma)| \text {. }
$$

${ }^{1}$ This work was sponsored in part by the U. S. Air Force. 
For each $\sigma$ in $S$, the translation mappings $R_{\sigma}$ and $L_{\sigma}$ of $C(S)$ into itself are defined by

$$
R_{\sigma} f(\tau)=f(\tau \cdot \sigma), \quad L_{\sigma} f(\tau)=f(\sigma \cdot \tau), \quad \text { all } \tau \text { in } S .
$$

A linear subspace $D$ of $C(S)$ that is invariant under all right and left translations, that contains the function 1 and that is closed under complex conjugation is called an amenable subspace (see [2]) of $C(S)$ if it possesses a two-sided invariant mean; i.e. an element $m$ in the dual $D^{*}$ of $D$ that satisfies $\langle m, 1\rangle=1,\langle m, f\rangle \geqq 0$ if $f \geqq 0$ and

$$
\langle m, f\rangle=\left\langle m, R_{\sigma} f\right\rangle=\left\langle m, L_{\sigma} f\right\rangle
$$

for all $f$ in $D$ and $\sigma$ in $S$, where $\langle\cdot, \cdot\rangle$ is the pairing between $D^{*}$ and $D$. The semigroup $S$ is itself called amenable if $C(S)$ is an amenable subspace of $C(S)$. If $S$ is commutative or is a solvable group, it is amenable; for further sufficient conditions for amenability, see [2].

2. Direct sum decompositions. In this section $B$ is a Banach space and $S$ a fixed uniformly bounded semigroup of operators on $B$ containing the identity operator. $S$ and its weak operator closure $\bar{S}$ are topological semigroups in the sense of $\$ 1$ when supplied with the weak operator topology. A subset $J$ of $S$ is called an ideal if $T J \subset J$ and $J T \subset J$ for all $T$ in $\bar{S}$. If $S$ is w.a.p., $\bar{S}$ will be compact and contain a unique minimal closed ideal $K_{\bar{S}}$.

Let $C_{B}(S)$ be the smallest subalgebra of $C(S)$ closed under complex conjugation and containing the function 1 and all functions $f$ which are of the form

$$
f(T)=\langle T x, y\rangle
$$

for some $x$ in $B$ and $y$ in its dual $B^{*}$.

$B_{p}$ is defined to be the closed linear subspace of $B$ spanned by those finite dimensional $S$-invariant subspaces of $B$ on which, relative to a suitable choice of basis, $S$ acts as unitary operators. If $S$ is a group, $B_{p}$ can be described more simply as the closed linear subspace of $B$ spanned by those $x$ in $B$ with orbit $O(x)$ finite dimensional. If $S$ is a commutative semigroup, $B_{p}$ can also be described more simply as being the closed linear subspace of $B$ spanned by those $x$ having $O(x)$ one-dimensional and which furthermore satisfy

$$
T x=\lambda_{T} x, \quad\left|\lambda_{T}\right|=1,
$$

for all $T$ in $S . B_{p}$ is the space of vectors almost periodic under $S$ in the terminology of [8]. $B_{0}$ is defined to be the set of all $x$ in $B$ for which 0 is in the weak closure of the orbit $O(x)$. 
TheOREM 1. Assume that $S$ is weakly almost periodic. Then the following are equivalent (with or without parenthesized terms):

1. $C_{B}(S)$ is an amenable subspace of $C(S)$.

2. $K_{\bar{S}}$, the minimal closed ideal of $\bar{S}$, is a (compact topological) group.

3. $B_{0}$ is a closed (S-invariant) linear subspace of $B$ and $B$ is the direct sum of $B_{0}$ and $B_{p}$.

If these conditions are satisfied and furthermore $S$ is almost periodic,

$$
\lim _{T \in S} T x=0
$$

in the norm topology for all $x$ in $B_{0}$.

The last statement of the above is to be interpreted to mean that for each $\epsilon>0$ there is a $T_{0}$ in $S$ for which $\left\|T_{0} T x\right\|<\epsilon$ and $\left\|T T_{0} x\right\|<\epsilon$ for all $T$ in $S$.

As a consequence of Theorem 1 , if $S$ is w.a.p. and an amenable semigroup, assertions 2 and 3 of Theorem 1 are valid. That assertion 3 holds for commutative $S$ and $B$ reflexive is the main result of Jacobs [8]. The equivalence of 1 and 2 is related to a result of Rosen in $[11]$.

Theorem 2. Assume that $S$ is weakly almost periodic, $\|T\| \leqq 1$ for all $T$ in $S$ and furthermore $B$ and its dual $B^{*}$ are strictly convex. Then assertions 1, 2 and 3 of Theorem 1 are valid.

This extends the main result of Jacobs in [9]. A fact crucial for the proofs of Theorems 1 and 2 is the result of Ellis [5] which guarantees that a group having a compact topology in which multiplication is separately continuous is a topological group; i.e. has multiplication jointly continuous and inversion continuous.

3. Weakly almost periodic functions. Let $S$ be a topological semigroup. $W(S)$, the space of weakly almost periodic functions on $S$, is defined to be the (automatically closed) linear subspace of $C(S)$ consisting of all $f$ having $\left\{R_{\sigma} f: \sigma \in S\right\}$ conditionally compact in the weak topology of $C(S)$ (the corresponding definition involving left translates is equivalent; this is Proposition 7 of [7]). The restrictions of the operators $R_{\sigma}$ to the Banach space $W(S)$ form a semigroup that is w.a.p. in the sense of $\$ 1$ so the results of $\$ 2$ apply. The weak operator closure $S^{w}$ of this semigroup is called the weakly almost periodic compactification of $S$. This is justified by the following:

THEOREM 3. The homomorphism $\sigma \rightarrow R_{\sigma}$ of $S$ into $S^{w}$ induces $a$ map $C\left(S^{w}\right) \rightarrow C(S)$ that is an isomorphism of $C\left(S^{w}\right)$ onto $W(S)$.

Thus in particular each weakly almost periodic function $f$ on $S$ is a 
composite $g \circ \Phi$ where $\Phi$ is the continuous homomorphism $\sigma \rightarrow R_{\sigma}$ of $S$ into the compact topological semigroup $S^{w}$ and $g$ is a continuous function on $S^{w}$. On the other hand, if $S^{\prime}$ is any compact topological semigroup, $\Phi^{\prime}: S \rightarrow S^{\prime}$ a continuous homomorphism and $g$ a continuous function on $S^{\prime}$, the composite $g \circ \Phi^{\prime}$ will be weakly almost periodic on $S$. This shows that compact topological semigroups play the same central role in the theory of weakly almost periodic functions as do compact topological groups in the theory of almost periodic functions on groups (for the group case see [12]).

We shall say that the topological semigroup $S$ is weakly amenable if $W(S)$ is an amenable subspace of $C(S)$; i.e. if there is a 2 -sided invariant mean on $W(S)$.

In the notation of $\S 2, W(S)_{p}$ is the closed linear subspace of $W(S)$ spanned by those functions that are coefficients of finite dimensional unitary representations of $S$, and $W(S)_{0}$ consists of all functions $f$ in $W(S)$ that have 0 in the weak closure of their set of right translates $\left\{R_{\sigma} f: \sigma \in S\right\}$.

As a consequence of Theorem 1

THEOREM 4. Let $S$ be a topological semigroup. Then the following are equivalent:

1. $S$ is a weakly amenable semigroup.

2. $K_{S w}$, the minimal closed ideal of $S^{w}$, is a compact topological group.

3. $W(S)_{0}$ is a closed linear subspace of $W(S)$ and $W(S)$ is the direct sum of $W(S)_{0}$ and $W(S)_{p}$.

In the case that $S$ is weakly amenable, the 2 -sided invariant mean on $W(S)$ is unique, and can be identified, by means of the isomorphism of Theorem 3, with Haar measure on the compact group $K_{S w}$. Thus Fourier analysis in $W(S)$ with respect to this mean can be identified with Fourier analysis with respect to Haar measure on $K_{S w}$ and in this way most of the results in [3] concerning weakly almost periodic functions extend to our context. Finally $W(S)_{0}$ can be characterized in this case as consisting of those $f$ in $W(S)$ having the mean of $|f|^{2}$ equal to 0 ; thus assertion 3 of Theorem 5 is seen to be the extension to our situation of the result announced by Eberlein in $[4]$.

4. Almost periodic functions. Let $S$ be a topological semigroup. $A(S)$, the space of almost periodic functions on $S$, is defined to be the closed linear subspace of $C(S)$ consisting of all $f$ having $\left\{R_{\sigma} f: \sigma \in S\right\}$ conditionally compact in the norm topology of $C(S)$. The restrictions of the operators $R_{\sigma}$ to the Banach space $A(S)$ form a semigroup that 
is a.p. in the sense of $\$ 1$ so the results of $\$ 2$ again apply. The strong operator closure $S^{s}$ of this semigroup (which is compact and thus identical with its weak operator closure) is called the almost periodic compactification of $S$.

As in Theorem 3, the map $\sigma \rightarrow R_{\sigma}$ induces an isomorphism of $A(S)$ with $C\left(S^{s}\right)$ and the appropriate modifications of the comments following Theorem 3 also are valid in this situation. Furthermore in this case the multiplication in $S^{8}$ is jointly continuous, and if $S$ is a group, $S^{s}$ will also be a group that can be identified with the usual almost periodic compactification (see [12]).

Let us now restrict our attention to commutative $S$. A semicharacter $\chi$ of $S$ is a complex valued function on $S$ satisfying

$$
\chi(x \cdot y)=\chi(x) \chi(y), \quad|\chi(x)| \leqq 1, \quad \text { all } x, y \text { in } S .
$$

It is easy to check that the closed linear subspace of $C(S)$ spanned by the semicharacters of $S$ is contained in $A(S)$. We shall say that the approximation theorem is valid for $S$ if this subspace is all of $A(S)$. That the approximation theorem is valid if $S$ is a group is the fundamental theorem of the theory of almost periodic functions on groups and is an immediate consequence of the existence of a compactification and the Peter-Weyl theorem. In our case no parallel of the latter is available and thus the existence of the compactification is not immediately helpful in general. And the approximation theorem is actually false for many semigroups, even for subsemigroups of groups in the discrete topology. (Let $S$ be all lattice points $(m, n)$ in the plane with $m>0$ or $m=n=0$; then an arbitrary bounded function $f$ on $S$ with $f(m, n)=0$ for $m>1$ is in $A(S)$ while all but two of the semicharacters of $S$ are exponentials). However, by special, and in some cases rather complicated arguments involving the structure of $S^{s}$, the approximation theorem can be established for certain semigroups.

The following is a partial list of the topological semigroups for which we have established the approximation theorem.

1. Cones in Euclidean $n$-space, usual topology;

2. Finitely generated subsemigroups of abelian groups, discrete topology;

3. Any closed subsemigroup $S$ of an abelian topological group $G$ with $G=S \cup(-S)$, relative topology;

4. Non-negative elements in a totally ordered abelian topological group (when closed), relative topology;

5. Totally ordered set under sup, discrete topology.

Furthermore the class of topological semigroups for which the ap- 
proximation theorem holds is closed under the formation of products; this is a consequence of the Stone-Weierstrass theorem and the fact that the almost periodic compactification of a product is the product of the compactifications. This last fact is false for the w.a.p. compactification.

\section{BIBLIOGRAPHY}

1. H. Anzai and S. Kakutani, Bohr compactifications of a locally compact abelian group I and II, Proc. of the Imperial Academy, Tokyo, vol. 19 (1943) pp. 476-480; 533-539. 544.

2. M. M. Day, Amenable semigroups, Illinois J. of Math. vol. 1 (1957) pp. 509-

3. W. F. Eberlein, Abstract ergodic theorems and weakly almost periodic functions, Trans. Amer. Math. Soc. vol. 67 (1949) pp. 217-240.

4. - Spectral theory and harmonic analysis, Proceedings of the Symposium on Spectral Theory and Differential Problems, Oklahoma Agricultural and Mechanical College, 1951, pp. 209-220.

5. R. Ellis, Locally compact transformation groups, Duke Math. J. vol. 24 (1957) pp. 119-126.

6. M. Fréchet, Les fonctions asymptotiquement presque-periodiques, Rev. Sci. vol. 79 (1941) pp. 341-354.

7. A. Grothendieck, Critères de compacité dans les espaces fonctionnels generaux, Amer. J. Math. vol. 74 (1952) pp. 168-186.

8. K. Jacobs, Ergodentheorie und fastperiodische Funktionen auf Halbgruppen, Math. Z. vol. 64 (1956) pp. 298-338.

9. - Fastperiodizitätseigenschaften allgemeiner Halbgruppen in Banach Rüumen, Math. Z. vol. 67 (1957) pp. 83-92.

10. W. Maak, Fastperiodische Funktionen auf Halbgruppen, Acta Math. vol. 87 (1952) pp. 33-57.

11. W. G. Rosen, On invariant means over compact semigroups, Proc. Amer. Math. Soc. vol. 7 (1956) pp. 1076-1082. 1940.

12. A. Weil, L'integration dans les groupes topologiques et ses applications, Paris,

STANFORD UNIVERSITY AND

University OF NOTRE DAME 